\title{
Optimization of Communication to Improve the Medication Process in Nursing Home Care - a Qualitative Evaluation
}

Nadja Nestler ( $\nabla$ nadja.nestler@pmu.ac.at)

Institute of Nursing Science and Practice https://orcid.org/0000-0002-7342-0394

Angelika Mahlknecht

Paracelsus Medical Private University: Paracelsus Medizinische Privatuniversitat

Ulrike Bauer

Paracelsus Medical Private University: Paracelsus Medizinische Privatuniversitat

\section{Laura Krisch}

Paracelsus Medical Private University: Paracelsus Medizinische Privatuniversitat Juergen Osterbrink

Paracelsus Medical Private University: Paracelsus Medizinische Privatuniversitat

Maria Flamm

Paracelsus Medical Private University: Paracelsus Medizinische Privatuniversitat

\section{Research article}

Keywords: Interprofessional cooperation, interprofessional communication, medication process, health information technology

Posted Date: January 20th, 2021

DOl: https://doi.org/10.21203/rs.3.rs-149559/v1

License: (c) (i) This work is licensed under a Creative Commons Attribution 4.0 International License. Read Full License 


\section{Abstract}

Background: Cooperation and communication are central factors for a safe medication process, especially residents of nursing homes, due to their multimorbidity, and hence, their need of numerous drugs. An interprofessional team is needed to handle potential problems of the medication process, but as several studies confirm, communication and cooperation within the medication process allow for improvement. This study aims to understand functioning and malfunctioning aspects of communication and cooperation within the medication process, to use these results to optimize the communication and coordination between the involved professional groups, and to examine changes after intervention.

Methods: This qualitative study is part of an interventional study. Involved professional groups of the medication process were interviewed before and after an intervention to evaluate experiences regarding communication, cooperation, as well as with the intervention overall. Six group discussions and two expert interviews with general practitioners, nurses of nursing homes and pharmacists were recorded and transcribed. Data analysis was done via qualitative content analysis to examine communication and cooperation of the professional groups. A derived coding tree was developed, output from codes was used to sort data into themes, and quotations were selected that exemplified themes for inclusion in this manuscript.

A combined intervention was implemented that included an education program concerning medication safety and an online communication tool.

Results: Responsibility and availability are two central aspects for efficient cooperation and communication. Problems are mostly system-related due to the necessity to use traditional ways of communication. The usage of a new online communication platform enables a regular medication review and an independence from personal attendances of all professional groups.

Conclusion: An interprofessional education program as well as the implementation of an online communication tool overcame barriers of communication and cooperation, systematized the medication process, and allowed for independent working routines of the professional groups.

Trial registration: DRKS Data Management, ID: DRKS00007900

\section{Background}

Medication safety represents a major topic within the interprofessional collaboration in nursing homes (NHs). Many older people, especially in NHs, have increased healthcare needs because of chronic illness (1). $81 \%$ of women and $74.3 \%$ of men aged 75 years and older described a self-reported chronic condition (2). Chronic illness often introduces the necessity of long-term medication and polypharmacy, which increases the frequency of medication errors (3). An interprofessional dialogue is needed to minimize risks within the medication process and optimize medication management (4). However, different approaches regarding work organization of the involved healthcare professionals (general practitioners 
(GPs), nurses in NHs and pharmacists) lead to challenges in successful communication. Studies show insufficient interprofessional cooperation and communication within the medication process $(3,5)$. Except in cases of health emergencies, the professional groups have different timeslots to communicate with the others leading to barriers. Due to unsynchronized, traditional ways of communication like telephone or fax (6) and barriers of availability, direct contacts are often difficult. This often causes problems in situations of disagreement, when the medication process and the necessities are evaluated differently (1).

For an optimization of the cooperation and communication between the professional groups, it is useful to apply modern communication channels to synchronize communication, which improves the medication process and optimizes the residents' situation $(1,5)$.

The project InTherAKT (Initiative Therapiesicherheit in der Altenhilfe durch Kooperation und Teamwork Initiative for medication safety in long-term care via cooperation and teamwork) aimed to optimize medication safety in NHs. Therefore, after educating the participating professional groups, the medication process was structured by implementing a specific online communication tool including a systematic therapy-check.

\section{Methods}

The here presented qualitative part of a single-arm interventional study (2014-2017) with an explanatory sequential design evaluated the experiences of communication and cooperation of the different professional groups in the field of medication safety in nursing homes before and after an intervention. The quantitative results have been published separately (7). Therefore, this article mainly describes the qualitative methods. Full methodical details are explained in the published study protocol (8).

Healthcare professionals operating in NH care (GPs, nurses, pharmacists) in a medium-sized city in Germany (Münster) were involved in the study as a convenience sample.

Inclusion criteria were:

- GPs: medical treatment of residents of nursing homes.

- NHs: treated residents of participating GPs. Nurses with a 3-year state approved training

- Pharmacists: an existing drug supply contract with the NHs, where the participating residents lived.

\section{Collection of data}

Study participants were informed in detail about the project by the research team and gave their written consent to participate in the interview. We conducted semi-structured, guided group discussions for each professional group before (June-July 2015) and after (November-December 2016) the intervention phase, which took place in a meeting-room of a public building. Each person of the participating institutions was invited via post or e-mail and gave her/his written consent before the interview. The 
group discussions were conducted by two research team members (1. moderation, non-directive style, 2 . observation, field notes and ad-hoc questions). The used interview guideline was conducted for this study (supplementary file) and served as an orientation and was applied for all professional groups. It included a discussion impulse as well as specific topics about:

- experiences of the healthcare professionals regarding cooperation and communication with the other groups

- their own tasks in the medication process

- their perceived needs for change

- approaches to possible solutions

- their satisfaction regarding the cooperation with the other professional groups

- additionally, at the end of the study: the practicability of the online platform of the research project for everyday practice

Moreover, the participants had the possibility to bring in other topics or to deepen certain topics.

Prior to each interview, characteristics of the participants were collected via a brief standardized questionnaire (gender, time of professional affiliation, period of employment in NH/pharmacy/GP office).

\section{Data analysis}

The audiotaped and transcribed interviews were analyzed via qualitative content analysis using a coding technique (9) with a derived coding tree before and after the intervention (Table 1). Deductive category application using the semi-structured interview guideline was combined with inductive categories from the data. The software program was used to assist analysis and to manage data. All coded text passages were paraphrased and generalized by two team members who systematized them thematically and summarized their content. For publication, all citations were translated from German to English and the English text obtained from translation was compared to the original German interview text to assess and guarantee the original meaning. The trustworthiness of qualitative data is assured by exploring the way of data collection and analysis. Citations of the interviews were used to support the transparency. 


\begin{tabular}{|c|c|}
\hline Main Category & Subcategory \\
\hline \multirow[t]{3}{*}{ Experience regarding cooperation } & $\begin{array}{l}\text { occasion of cooperation to the topic drug } \\
\text { treatment }\end{array}$ \\
\hline & working cooperation \\
\hline & cooperation problems \\
\hline \multirow[t]{3}{*}{ Experience regarding communication } & $\begin{array}{l}\text { occasion of communication to the topic drug } \\
\text { treatment }\end{array}$ \\
\hline & working communication \\
\hline & communication problems \\
\hline \multirow{3}{*}{$\begin{array}{l}\text { Own tasks for warranty of the drug treatment } \\
\text { safety of }\end{array}$} & GP's \\
\hline & nurses \\
\hline & pharmacists \\
\hline \multirow[t]{3}{*}{ Need for changes to ensure drug therapy safety } & -- \\
\hline & -- \\
\hline & - \\
\hline \multirow[t]{3}{*}{ Satisfaction with the cooperation with } & GP's \\
\hline & nurses \\
\hline & pharmacists \\
\hline
\end{tabular}

Legend: GPs $=$ General Practitioners

\section{Intervention}

The combined intervention was conducted by the interprofessional project team (pharmacists, nurses, physicians) and comprised two steps:

- education for all participating professionals to expand their knowledge about medication safety in older adults. The education consisted of a face-to-face part with all professional groups and a following profession-specific online-education covering the main topics of drug safety in older adults (drug-drug and drug-disease interactions, medication errors, pharmacovigilance, polypharmacy, potentially inappropriate prescriptions, legal aspects of drug therapy, strategies to enhance interprofessional cooperation) (8). Finally, all participants had an on-site meeting to instruct them on the InTherAKT-online platform (I-oP). 
- systematic therapy-check (Fig. 1) via an online-communication tool developed for the study, called InTherAKT-online platform (I-oP). The scope of the I-oP was to introduce the process itself, integrate all professional groups as well as visualize all resident information necessary for the medication process (age, gender, corresponding NH/GP/pharmacist, diagnoses, allergies/drug intolerances, height/weight, relevant laboratory values, physician visits/hospitalizations) and medication-related data (brand name, International Nonproprietary Name, indication, dosage, form/time of administration, indications for administration/monitoring, regular/as-needed medication, prescriber, date of first prescription). In addition, nurses documented occurring residents' symptoms in the l-oP to inform all professional groups. Data recording of all residents as well as the routine documentation in the NHs was conducted by study assistants (trained nursing students with an undergraduate nursing degree), not by the nurses of the participating $\mathrm{NHs}$ because of their high routine workload, who supervised the documentation and were involved by dialogue with GPs. The design of the I-oP was provided by the software-engineering company smart-Q (Germany).

\section{Results}

Before the intervention, one group discussion (GD) with 10 GPs was conducted, one with 5 pharmacists and at the same time two GDs with 24 nurses from $10 \mathrm{NHs}$ (duration 45-75 minutes).

After the intervention, one GD with 6 GPs and one with 5 pharmacists were performed. No GD was feasible with the nurses after the intervention. 2 nurses were interviewed separately with the same interview guideline as for the GDs. The interviewed persons were partly the same at both times of measurement. All post-interviews lasted from 15 to 25 minutes. Table 2 shows the characteristics of all participants.

Table 2

Characteristics of the interview participants

\begin{tabular}{|c|c|c|c|c|c|c|c|}
\hline & & Nurses & & GP's & & Pharmacists & \\
\hline & & $\begin{array}{l}\text { pre } \\
\text { inter- } \\
\text { vention } \\
(n=24)\end{array}$ & $\begin{array}{l}\text { post } \\
\text { inter- } \\
\text { vention } \\
(n=2)\end{array}$ & $\begin{array}{l}\text { pre } \\
\text { inter- } \\
\text { vention } \\
(n=10)\end{array}$ & $\begin{array}{l}\text { post } \\
\text { inter- } \\
\text { vention } \\
(n=6)\end{array}$ & $\begin{array}{l}\text { pre } \\
\text { intervention } \\
(n=5)\end{array}$ & $\begin{array}{l}\text { post } \\
\text { inter- } \\
\text { vention } \\
(n=5)\end{array}$ \\
\hline Female & $\mathrm{N}(\%)$ & $\begin{array}{l}18 \\
(75.0)\end{array}$ & $2(100)$ & $3(30.0)$ & $3(50.0)$ & $3(60.0)$ & $\begin{array}{l}4 \\
(80.0)\end{array}$ \\
\hline $\begin{array}{l}\text { number of years } \\
\text { of employment }\end{array}$ & $\begin{array}{l}\text { Mean } \\
\text { (SD) }\end{array}$ & $\begin{array}{l}18.5( \pm \\
9.0)\end{array}$ & $\begin{array}{l}38.5( \pm \\
13.5)\end{array}$ & $\begin{array}{l}18.1 \\
( \pm 7.7)\end{array}$ & $\begin{array}{l}23.0 \\
( \pm 13.6)\end{array}$ & $17.2( \pm 8.3)$ & $\begin{array}{l}17.2 \\
( \pm 9.9)\end{array}$ \\
\hline
\end{tabular}


The themes discussed before and after the intervention could be assigned to a total of eight categories (Fig. 2). In this article, the central themes 'cooperation and communication between the professional groups', 'change requirements at the beginning of the project' and 'changes due to the project' are presented. The perceptions of each professional group are offered consecutively, common perceptions are presented together.

\section{Main results of the interviews}

\section{Cooperation and communication between the professional groups}

A functioning cooperation and communication at the beginning of the project was perceived when GPs took responsibility for the resident's prescribed medication and checked it regularly. High numbers of treated residents, high frequency of visits during off-office-hours as well as regular visits in the NHs by the GP were perceived as responsible behavior by the nurses.

"There are GPs who come for rounds regularly every 14 days or every week, with whom you can work well because not only [the nurses] have questions, but [the GPs] also asks how his patients are doing." (GD nurses_gr., 502).

GPs mentioned the nurses' knowledge of drugs as important for a good cooperation as well as the organization of information transfer, e. g. about changes in medication (prescription of medical specialists) by faxing the updated medication plan of residents to the GPs.

All professional groups described the importance of the reachability of the others for a well working cooperation and communication. The nurses and GPs defined the accessibility of the pharmacy, especially outside opening hours, and a swift delivery of medications for an acute treatment in urgent cases as beneficial. The nurses also described support by the pharmacists in form of medication reviews in NHs and regular trainings by pharmacists as helpful as well as the proximity of local pharmacies for a better coordination of drug management. The pharmacists pronounced only rare contacts to GPs. They called a cooperation well-functioning when they informed the GPs via fax about medication problems.

Nurses described problems in the cooperation with the GPs, because of differences in the prescription of medication. Sometimes GPs might not prescribe special medications, like neurological drugs, or fail to recognize the resident-specific limitations in application, such as the prescription of tablets for persons with a feeding tube.

“... You don't know if the GP always knows what another specialist has prescribed. They are often quick on their visits and often rarely on the spot. If the GPS offices are distant away, they [the GPS] may visit every three months. During the visits by the pharmacy, it was noticeable that medication was still available that had been discontinued for a long time or that there were also no needs for the medication. That's where you have to start." (GD pharmacists 1, 71-77). 
Another aspect raised by the GPs was the missing information about prescriptions ordered by medical specialists, so that they were usually unaware about the total current medication of the residents and they mentioned missing information about drug-drug interactions by the pharmacists. In contrast, the pharmacists described different reactions of the GPs when informing them about problems of medication management. They experienced that most of the GPs did not want to be informed by pharmacists.

The pharmacists complained that their expertise was not acknowledged - neither by the GPs nor by the nurses, so that they lacked information about changes of the residents' status, which could be helpful when reviewing possible medication problems.

For the GPs, an independent inspection of the resident's documentation was not possible, because they normally had no free access to the used electronic documentation systems in NHs, and had to rely on the nurses' access to the system, mostly without a regular contact person and with an organization-specific medication process. These circumstances inhibited GPs from frequently visiting a $\mathrm{NH}$.

"One often has the problem in the homes that one must look for the nursing staff often for hours. This keeps the doctor from going to the home. You have to go into a room to ring the bell and then it takes another ten minutes. The biggest problem is that the nurses are not there. (GD GPs 1, 99-112).

Moreover, GPs and nurses interpreted the medication process differently. For example, GPs and nurses valued a medication order via telephone differently with regards to legal rules.

"Many orders come by phone. Even if you are working correctly yourself, you always think that the doctor should take a look at the resident first. The remote diagnoses (should) become a little less. One also doesn't know whether most [GPS] would take time for the documentation, it is spoken faster than written. (GD nurses_yel., 238-240).

\section{Change requirements at the beginning of the project}

All interviewed persons recognized the necessity of changes in cooperation and communication concerning the medication process, e.g. the need for better technical facilities in the face of medical prescription and the need to involve all professional groups in the medication process to cover different angles. The nurses demanded the GPs to show more responsibility for drug therapy safety and the whole medication process, especially regarding regular visits in the $\mathrm{NHs}$ and responsible prescription of drugs.

"It would be nice if the doctors showed more responsibility, did not settle everything by telephone if possible, but looked at the residents on the spot and receive their own impression and did not always have a blind faith that the nurses would pass on everything correctly. They should also show responsibility to look at the medication prescribed by specialists together, because they are the family doctors and responsible for the residents. However, they often say that they have nothing to do with it, which is perceived as difficult. (GD nurses_yel., 220). 
In turn, the GPs expressed a better assumption of responsibilities by the nurses for the therapy observation as well as for the transfer of information to the GPs. The pharmacists correspondingly saw the need for changes in nursing strategies to improve the medication process by integration of medication safety into regular nursing activities. They also wanted to have regular visits with nurses and GPs.

\section{Changes due to the project}

After the intervention, all professional groups described different experiences as a result of the project. The GPs felt well-trained by the online education and experienced the networking as important. The pharmacists felt a higher acknowledgement of their expertise by the GPs and the nurses. After the intervention, they were contacted more often by the GPs seeking their advice and the communication was improved and more partner-like.

After the medication review of the pharmacists, the nurses observed a change in the GPs' behavior in the form of increased responsibility and communication improved further during the project. More regular visits, a standardized exchange of medication and differences in the medication documentation between $\mathrm{NHs}$ and GPs offices, that became apparent during the intervention, sensitized the GPs and nurses to drug therapy safety because of the intervention. The GPs felt obliged to reflect on the medication plans of the residents following the medication review of the pharmacists. The onsite educational part was rated as beneficial as it created a new habit of periodically checking all medications.

"Just the fact that you really thought about it again and checked everything again. This also led to the discontinuation of medication. If you actually live what you have learned in advance during the courses [online modules] and perhaps bring in regularity, this awareness of the problem earns a lot." (GD GP 2, 298-304).

The use of the online platform was a central topic after the intervention. It was described as a helpful device and a positive way to communicate. The pharmacists identified the benefit of electronic networking, because it enabled independence from personal attendances of all professional groups. The nurses were aware of the residents' current situation by focusing on the therapy observation and the documentation of the other health care professionals in the tool. The GPs found the regular medication review by pharmacists supportive. The pharmacists experienced that the GPs responded to their inquiries and warnings. Problems because of local distances to the GPs office were reduced by the online platform. Since in German NHs medication reviews are usually not carried out and communicated, the pharmacist's found it necessary to continue this practice after the intervention, e. g. regular communication with the GPs.

A change concerning the cooperation was the nurses' reflection about their inadequate knowledge about storage and administration of drugs. At the same time, the GPs described the necessity of getting comprehensive information from nurses about possible problems with the administration. This created a gap in the communication flow, which could be closed by further education and communication. 
All professionals noted an improved communication because of a greater awareness regarding drug safety. Direct communication was experienced as positive. However, the pharmacists also problematized their own role because of scrutinizing the GPs by conducting the medication review.

\section{Discussion}

The interviews with several professional groups aimed to evaluate the experiences of each group regarding the cooperation and communication with the other groups about the medication process in $\mathrm{NHs}$. Nurses of nursing homes and pharmacists as well as GPs were involved in the study and the differences between the perceptions of the professional groups before and after the intervention could be compared.

The underlying assumption was that an increase in drug therapy safety for residents of NHs requires an optimization of cooperation and communication between the involved healthcare professionals. The systemic and technical prerequisites seemed to be important (1) as well as a systematization of the cooperation and communication processes (10). In addition, the mutual acceptance of the professional groups as partners in the medication process and the insight into the need for cooperation to ensure qualitative medication therapy appeared to be essential (11). Our study confirms these findings.

\section{Systematization of cooperation and communication}

At the beginning of the project, all professional groups described problems in cooperation and communication. As in other studies $(10,12)$, these problems were mainly system-related, e. g. traditional hierarchical roles in collaboration and traditional ways of communication. It became clear that different focal points of work and different assessments in the process often caused problems. Technical barriers in communication as well as a lack of infrastructure and technical support creating an inadequate drug therapy safety were described in the literature $(5,6)$. These system-related cooperation and communication problems, which were also mentioned in our study, were at least partially resolved, or improved in our project by introducing the I-oP as well as the face-to-face education. The work with the IoP made it possible to eliminate the communication difficulties caused by problematic accessibility. Weissenborn et al. found that the interprofessional communication of GPs and pharmacists did not depend on a systematization of communication alone, but individualization was also important for the improvement of the communication process (12). The use of the l-oP and the successful bridging of the communication difficulties, which was evaluated as helpful in our study, is a positive result since systematization led to individual communication processes by telephone or personal contacts. To know someone works beneficially for the interprofessional exchange also underlines the possibilities of systematized communication for individualization of the communication processes.

The face-to-face education supported the perception of the mutual professionalism, which also represents an explanation for the changed communication in the course of the project. Pharmacists' advices on medication could be communicated to the GPs via the I-oP and were experienced by the GPs as helpful for their own work processes as described by Weissenborn et al. (12). 
The nurses described the importance of regular GPs visits and the resulting commitment to coordination and better knowledge as a basis for good cooperation at the beginning and the end of the project. The importance of a good relationship between nurses and GPs to ensure quality of residents' care is described by the GPs in our study as elsewhere $(13,14)$.

\section{Optimization of documentation and its importance}

During the intervention, a divergence in documentation of the GPs offices and the NHs' documentation emerged that was adjusted due to the use of the I-oP. The intensified discussion described by nurses and GPs regarding the diagnoses of the individual resident and the comparison with the current medication revealed information gaps and the I-OP was helpful regarding alignment of documentation. In another study on drug therapy safety among nursing home residents (10), divergence in medication plan of GPs' offices and NHs were also cited as a reason for inadequate pharmacotherapy. In addition, at the beginning of our project not all occupational groups had access to relevant medication information of the residents. This problem was solved by using the I-OP. This is an aspect of drug therapy safety that has received little attention in research so far.

All professional groups addressed the problems in cooperation and communication between GPs and other medical specialists. This problem, which was also described in previous studies, was still present at the end of the project, not least because the work with the I-oP made the knowledge about specialist orders and the need for coordination more obvious (15).

\section{Changes by the project}

All professional groups described changes they attributed to the intervention. The use of the l-oP was assessed as positive, as it enabled a systematic communication without having to rely on the direct accessibility of others. The success of the use of technological solutions not only depends on these technologies themselves but also on the social relationship of the actors and cultural characteristics (1). This can also be illustrated by our results, because the participants reported that the meaningfulness of working with the online platform depended not only on the technical improvement of the platform, but also on the perception of mutual professionalism. Above all, however, the knowledge about the competences and tasks of the other groups had increased, and thus, openness towards the profession and competences of the other actors was achieved. This created an important prerequisite for effective cooperation to improve drug therapy safety $(10,12)$.

\section{Limitations}

Our study has some limitations. At first, we cannot assume that data saturation has been achieved, as we had only a limited count of GPs, pharmacists, and nurses available for data collection. Maybe other participants had different experiences, or the same intervention would lead to different results in other settings. Besides that, only two nurses agreed to an interview after intervention. The other nurses refused to participate in the interview because of their high workload. We had to change the method and 
conducted expert-interviews with the two nurses. We were aware of this change in methodology but did not want to completely exclude the experiences of the nurses and considered their findings important for the evaluation of the project. Yet we recognize that the here presented results may only report a small focus.

Due to the routine workload of the nurses, the documentation in the l-oP in the NHs was not conducted by the nurses themselves but by study assistants who were however always supervised by the nurses. We don't know whether and to which extent this actually poses a limitation for the effectiveness of the intervention. This can in future be remedied by integrating the I-oP into the primary documentation system of the NHs.

Furthermore, no member validation was conducted with the participants of the interviews.

\section{Conclusion}

The implementation of an online communication tool helped to overcome cooperation and communication barriers between the involved professional groups. It systematized the medication process and allowed an independent work of the different groups. Moreover, the face-to face education and personal meetings were important for improving communication within the medication process. Our results show that a combination of education and systematization of the communication is effective in enhancing the interprofessional exchange within the medication process in $\mathrm{NHs}$.

\section{Abbreviations}

$\mathrm{GP}=$ general practitioner

$\mathrm{NH}=$ nursing home

$\mathrm{I}-\mathrm{oP}=$ InTherAKT-online platform

InTherAKT = Initiative Therapiesicherheit in der Altenhilfe durch Kooperation und Teamwork - Initiative for medication safety in long-term care via cooperation and teamwork

$\mathrm{GD}=$ group discussion

$\mathrm{SD}=$ Standard Derivation

\section{Declarations}

\section{Ethics approval and consent to participate}

The study protocol was registered via the German Clinical Trials Register (ID: DRKS00007900) and approved by the responsible Ethics Committee of the Medical Association of Westphalia-Lippe and of the 
Westphalian Wilhelms University of Muenster on 2015-03-27 (approval number 2015-147-f-S). All participants of the study gave their written consent to participate.

\section{Consent for pubication}

Not applicable

\section{Availability of data and materials}

The dataset analyzed during the current study is available on request from the corresponding author.

\section{Competing interests}

The authors declare that they have no competing interests.

\section{Funding}

The study was granted by commercial funding (Grünenthal $\mathrm{GmbH}$, Germany), the city of Muenster (Germany) and the local government of Salzburg (Austria). None of the sponsors had any access or influence on conceptual design, collection, evaluation, or presentation of data.

\section{Authors' contribution}

- $\mathrm{NN}$ : (had) collected and interpreted the data of the article; she drafted and designed the article, wrote the main text, and revised the work.

- AM: was part of the data analysis group, had a substantial contribution to the conception of the article, she drafted the work critically for important intellectual content.

- UB: had a substantial contribution to the conception of the article and revised the work critically for important intellectual content.

- LK: was part of the data analysis group; she had a substantial contribution to the conception of the article, she drafted the work critically for important intellectual content.

- JO: was part of the data analysis group; he had a substantial contribution to the conception and design of the article and revised the work critically for important intellectual content.

- MF: was part of the data analysis group; she had a substantial contribution to the conception of the article, she drafted the work critically for important intellectual content.

All authors read and approved the manuscript.

\section{Acknowledgements}

Not applicable

\section{References}


1. Barr N, Vania D, Randall G, Mulvave G. Impact of information and communication technology on interprofessional collaboration for chronic disease management: a systematic review. Journal of Health Services Research \& Policy. 2017;22(4):250-7.

2. Robert-Koch-Institute. Prevalence of chronic illness. 2009. In: In contribution to the health monitoring of the federal government [Internet]. Berlin: Robert-Koch-Institute.

3. Grewe H, Blättner B. Medication safety in ambulatory long-term care and in institutions. Pflegewissenschaft. 2017;11/12:557-64.

4. Grundmann-Horst B, Rosenthal-Schleicher K, Meissner A. Interprofessional drug review in geriatric care, learning experiences of nurses and pharmacists. Pflegezeitschrift. 2017;70(10):50-2.

5. Agency-for-Healthcare-Research-and-Quality. Enabling Medication Management Through Health Information Technology2011 14.06.2019.

6. Bates DW. Health Information technology and Care Coordination: The Next Big Opportunity for Informatics? 2015. In: IMIA Yearbook of Medical Informatics [Internet].

7. Mahlknecht, A, Krisch L, Nestler N, Bauer U, Letz N, Zenz D, et al. Impact of training and structured medication review on medication appropriateness and patient-related outcomes in nursing homes: results from the interventional study InTherAKT. BMC Geriatrics. 2019;19:257.

8. Mahlknecht A, Nestler N, Bauer U, Schüßler N, Schuler J, Scharer S, et al. Effect of training and structured medication review on medication appropriateness in nursing home residents and on cooperation between health care professionals: the InTherAKT study protocol. BioMed Central Geriatrics [Internet]. 2017; 18(17):[24 p.].

9. Mayring P. Qualitative Conten Analysis. Basics and techniques. Weinheim, Basel.2015.

10. Schmiemann G, Dehlfing A, Pulst A, Hoffmann F. Medication safety in nursing home residents with renal insufficiency - Results of a qualitative study. The Journal of Evidence and Quality in Health Care. 2017;121:14-20.

11. Blondal AB, Jonsson JS, Sporrong SK, Almarsdottir AB. General practitioners' perceptions of the current status and pharmacists' contribution to primary care in Iceland. International Journal of Clinical Pharmacy [Internet]. 2017.

12. Weissenborn M, Haefeli WE, Peters-Klimm F, Seidling HM. Interprofessional communication between community pharmacists and general practitioners: a qualitative study. International Journal of Clinical Pharmacy. 2017;39:495-506.

13. Gunnarsdottir S, Clarke SP, Rafferty AM, Nutbeam D. Front-line management, staffing and nursedoctor relationships as predictors of nurse and patient outcomes. A survey of Icelandic hospital nurses. International Journal of Nursing Studies. 2009;46:920-7.

14. Tourangeau AE, Cranley LA, Jeffs L. Impact of nursing on hospital patient mortality: a focused review and related policy implications. Quality and Safety in Health Care. 2006;15:4-8.

15. Spinewine A, Swine C, Dhillon S, Franklin BD, Tulkens PM, Wilmotte L, et al. Appropriateness of use of medicines in elderly inpatients: qualitative study. British Medical Journal [Internet]. 2005:[1-5 pp.]. 
GP reviewed pharmacists suggestions, released the revised medication list to nurses

Pharmacist informed GP about results of review and possible problems in the medication, indicated concrete solutions

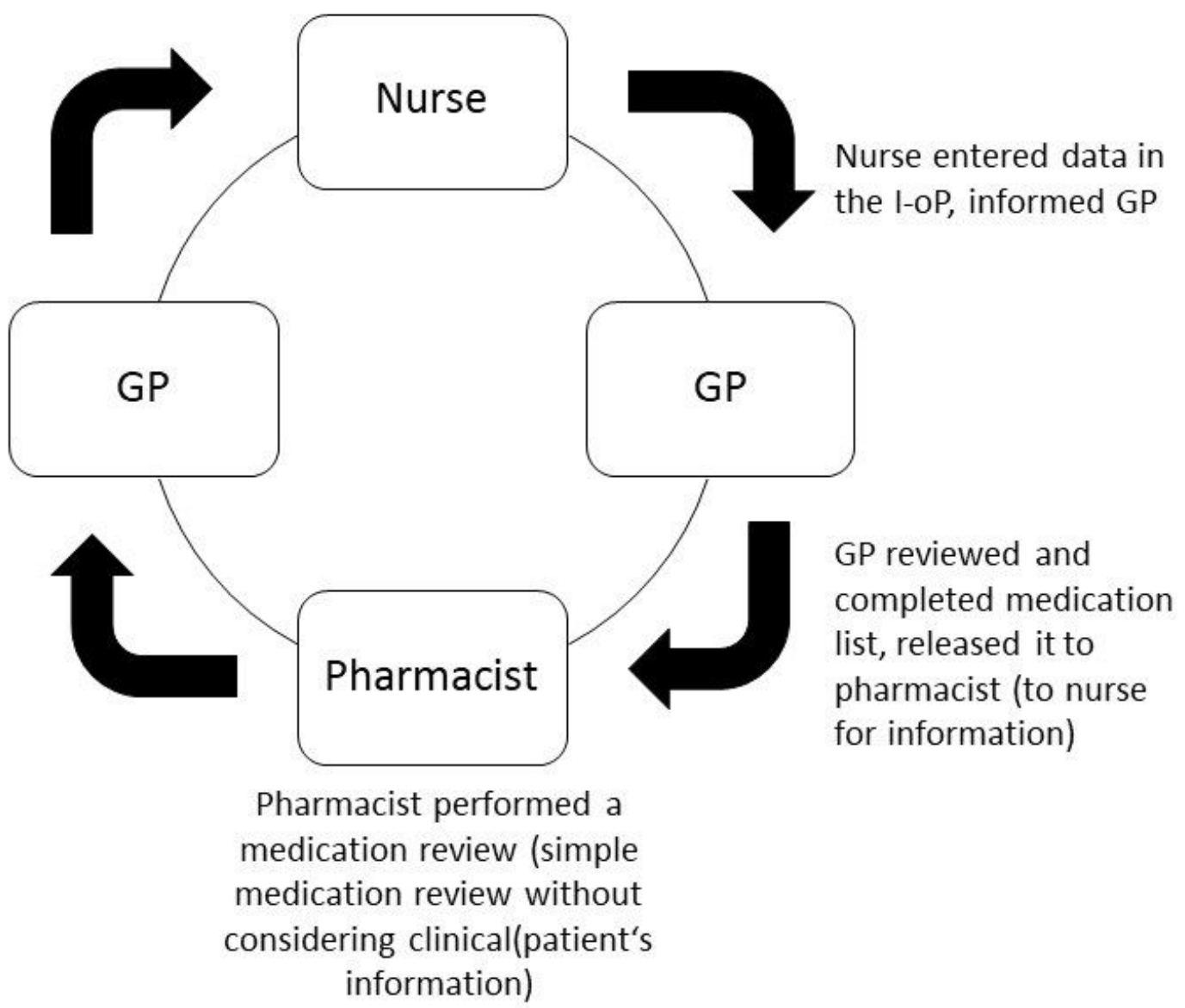

Figure 1

The therapy-check process 


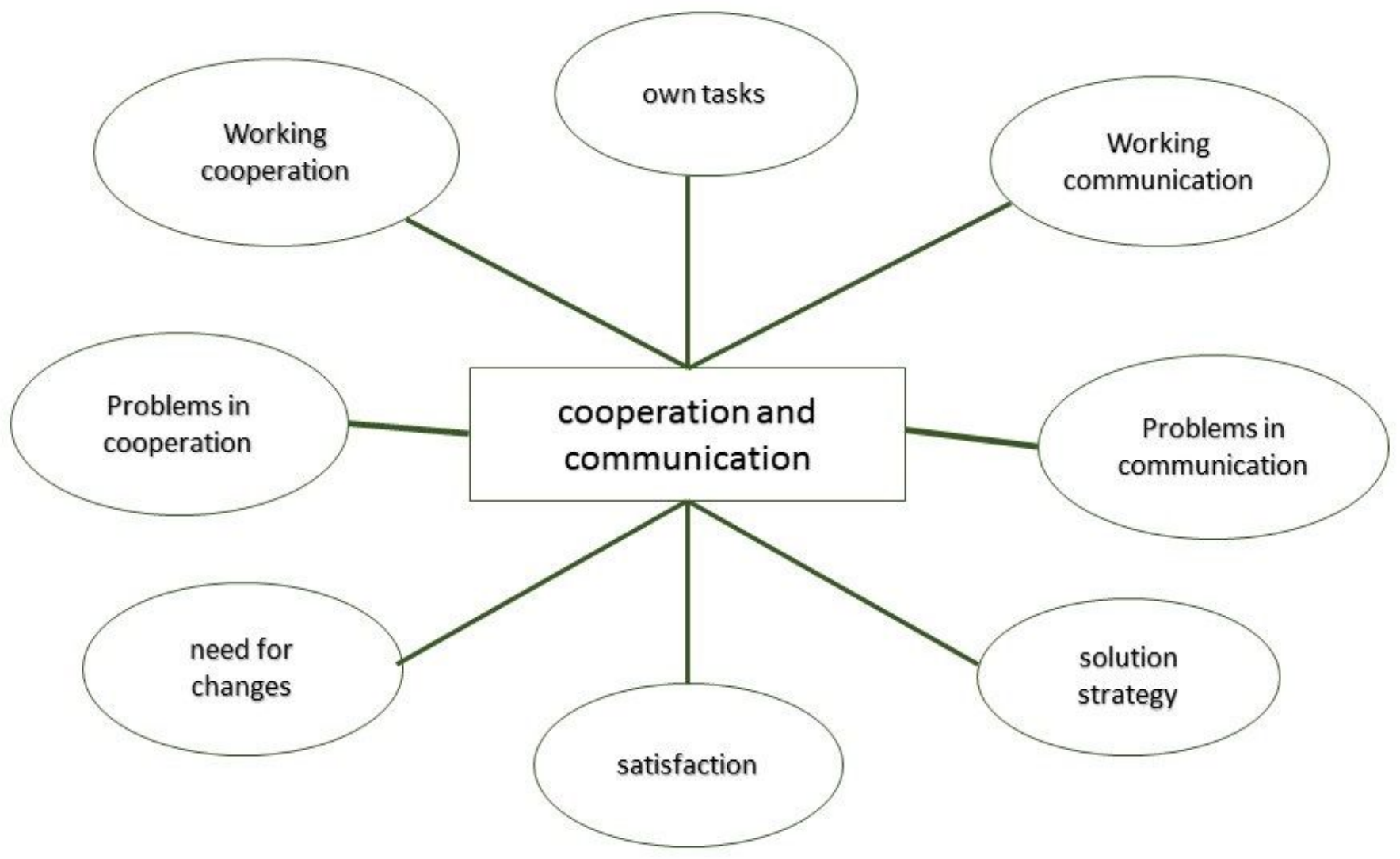

Figure 2

Main categories of the interviews

\section{Supplementary Files}

This is a list of supplementary files associated with this preprint. Click to download.

- COREQchecklist.pdf

- Interviewguide.docx 